\title{
Molecular emission from GG Carinae’s circumbinary disk ${ }^{\star}$
}

\author{
M. Kraus ${ }^{1}$, M. E. Oksala ${ }^{1}$, D. H. Nickeler ${ }^{1}$, M. F. Muratore ${ }^{2,3}$, M. Borges Fernandes ${ }^{4}$, A. Aret ${ }^{5,1}$, \\ L. S. Cidale ${ }^{2,3}$, and W. J. de Wit ${ }^{6}$ \\ 1 Astronomický ústav, Akademie věd České republiky, Fričova 298, 25165 Ondřejov, Czech Republic \\ e-mail: kraus@sunstel.asu.cas.cz \\ 2 Departamento de Espectroscopía Estelar, Facultad de Ciencias Astronómicas y Geofísicas, Universidad Nacional de La Plata, \\ Paseo del Bosque s/n, B1900FWA, La Plata, Argentina \\ 3 Instituto de Astrofísica de La Plata, CCT La Plata, CONICET-UNLP, Paseo del Bosque s/n, B1900FWA La Plata, Argentina \\ 4 Observatório Nacional, Rua General José Cristino 77, 20921-400 São Cristovão, Rio de Janeiro, Brazil \\ 5 Tartu Observatory, 61602, Tõravere, Tartumaa, Estonia \\ ${ }^{6}$ European Southern Observatory, Alonso de Cordova 3107, Vitacura, Santiago, Chile
}

Received 25 September 2012 / Accepted 8 November 2012

\begin{abstract}
Context. The appearance of the $\mathrm{B}[\mathrm{e}]$ phenomenon in evolved massive stars such as $\mathrm{B}[\mathrm{e}]$ supergiants is still a mystery. While these stars are generally found to have disks that are cool and dense enough for efficient molecule and dust condensation, the origin of the disk material is still unclear.

Aims. We aim at studying the kinematics and origin of the disk in the eccentric binary system GG Car, whose primary component is proposed to be a $\mathrm{B}[\mathrm{e}]$ supergiant.

Methods. Based on medium- and high-resolution near-infrared spectra we analyzed the CO-band emission detected from GGCar. The complete CO-band structure delivers information on the density and temperature of the emitting region, and the detectable ${ }^{13} \mathrm{CO}$ bands allow us to constrain the evolutionary phase. In addition, the kinematics of the $\mathrm{CO}$ gas can be extracted from the shape of the first ${ }^{12} \mathrm{CO}$ band head.

Results. We find that the $\mathrm{CO}$ gas is located in a ring surrounding the eccentric binary system, and its kinematics agrees with Keplerian rotation with a velocity, projected to the line of sight, of $80 \pm 1 \mathrm{~km} \mathrm{~s}^{-1}$. The CO ring has a column density of $(5 \pm 3) \times 10^{21} \mathrm{~cm}^{-2}$ and a temperature of $3200 \pm 500 \mathrm{~K}$. In addition, the material is chemically enriched in ${ }^{13} \mathrm{C}$, which agrees with the primary component being slightly evolved off the main sequence. We discuss two possible scenarios for the origin of the circumbinary disk: (i) nonconservative Roche lobe overflow; and (ii) the possibility that the progenitor of the primary component could have been a classical Be star. Neither can be firmly excluded, but for Roche lobe overflow to occur, a combination of stellar and orbital parameter extrema would be required.
\end{abstract}

Key words. stars: emission-line, Be - stars: early-type - supergiants - circumstellar matter - stars: individual: GG Car

\section{Introduction}

The Galactic emission line star GGCar was discovered more than a century ago (Pickering 1896; Pickering \& Fleming 1896). In addition to strong Balmer line emission, its optical spectrum displays numerous emission lines from permitted and forbidden transitions of predominantly low-ionized metals (e.g. Swings 1974; Marchiano et al. 2012). Strong line profile variation of emission features in the optical (e.g. Machado et al. 2004) and near-infrared (Morris et al. 1996) was reported, which might be interpreted as variability in the density structure of the wind. Furthermore, the spectral energy distribution shows a pronounced infrared excess, characteristic of emission from hot circumstellar dust (Allen 1973; Cohen \& Barlow 1983; Bouchet $\&$ Swings 1982). These reported characteristics, together with the temperature (Lopes et al. 1992) and luminosity estimates (McGregor et al. 1988), resulted in the classification of GGCar as a $\mathrm{B}[\mathrm{e}]$ supergiant $(\mathrm{B}[\mathrm{e}] \mathrm{SG}$; Lamers et al. 1998), although its supergiant status was long unclear due to highly uncertain distance estimates.

* Based on observations collected with the ESO VLT Paranal Observatory under programs 384.D-0613(A) and 088.D-0442(B).
GG Car displays radial velocity and light variability in both the optical and UV light curves, suggestive of an eclipsing binary system (Hernández et al. 1981; Gosset et al. 1984, 1985; Brandi et al. 1987). Based on radial velocity measurements of the blueshifted absorptions of the He I P Cygni profiles, Marchiano et al. (2012) recently refined the period of this eccentric binary system to $31.033 \pm 0.008 \mathrm{~d}$. In addition, these authors obtained orbital parameters for the two components, as well as improved stellar parameters for the primary, summarized in Table 1 . The mass of the primary and its temperature estimate confirm the supergiant status of GGCar. With this knowledge, the analysis of Marchiano et al. (2012) also provided the distance of $5 \pm 1 \mathrm{kpc}$ toward GGCar based on two independent methods. This distance estimate places GGCar in the vicinity of other evolved massive stars such as the luminous blue variables (LBVs) HR Car $(d=5 \pm 1 \mathrm{kpc}$; van Genderen et al. 1991; Hutsemékers \& van Drom 1991) and AGCar ( $d=6 \pm 1 \mathrm{kpc}$; Humphreys et al. 1989), but keeping in mind that distance estimates generally have high uncertainties.

Optical polarimetric (Gnedin et al. 1992; Klare \& Neckel 1977; Barbier \& Swings 1982) as well as interferometric (Borges Fernandes 2010) observations confirmed that the dust distribution is non-shperically symmetric, most probably located 
Table 1. Stellar parameters of the primary component of GG Car from Marchiano et al. (2012).

\begin{tabular}{ll}
\hline \hline$T_{\text {eff }}[\mathrm{K}]$ & $23000 \pm 2000$ \\
$L / L_{\odot}$ & $(2.6 \pm 1.0) \times 10^{5}$ \\
$R_{*}\left[R_{\odot}\right]$ & $32 \pm 8$ \\
$M\left[M_{\odot}\right]$ & $26 \pm 4$ \\
$i\left[^{\circ}\right]$ & $63 \pm 9$ \\
$d[\mathrm{kpc}]$ & $5 \pm 1$ \\
$E(B-V)[\mathrm{mag}]$ & $0.51 \pm 0.15$ \\
\hline
\end{tabular}

within a disk or disk-like structure. Preliminary analysis of the interferometric data deliver an inclination angle of about $60^{\circ}$, which excellently agrees with the inclination of the orbital plane of $54^{\circ}-72^{\circ}$ found by Marchiano et al. (2012). Furthermore, $\mathrm{H} \alpha$ spectropolarimetric studies of Pereyra et al. (2009) revealed a rotating disk-like structure. A disk is an ideal environment, not only for dust, but also for molecules, such as the very robust $\mathrm{CO}$ molecule. Emission in $\mathrm{CO}$ bands from GG Car has been reported by McGregor et al. (1988) and Morris et al. (1996).

Kraus (2009) reported on the detection of ${ }^{13} \mathrm{CO}$-band emission from GGCar based on a low-resolution $(R \simeq 1500)$ spectrum obtained with AMBER at the VLTI. The strength of the isotopic bands indicated that the circumstellar material is chemically enriched with processed material that must have been transported to the stellar surface and released into the environment by either strong non-spherically symmetric stellar winds or mass ejection events. The observed enhancement with ${ }^{13} \mathrm{C}$, however, strictly disagrees with theoretical model predictions of both rotating and non-rotating stellar models, and the disagreement could only be solved by the postulation of a higher mass (and thus luminosity) of GGCar than those reported in the literature. The new stellar parameters determined by Marchiano et al. (2012; see Table 1) moved the position of GG Car in the Hertzsprung-Russell (HR) diagram to a more appropriate place. Still, the proper determination of the amount of ${ }^{13} \mathrm{CO}$ and accordingly the ${ }^{12} \mathrm{C} /{ }^{13} \mathrm{C}$ ratio, which is an excellent tracer of the stellar evolutionary phase (Kraus 2009; Liermann et al. 2010), is lacking.

Probing the evolutionary stage of $\mathrm{B}[\mathrm{e}] \mathrm{SG}$ s is an important issue for understanding their mass-loss history, and hence the origin and shaping mechanism of their circumstellar environment resulting in the $\mathrm{B}[\mathrm{e}]$ phenomenon. For this, the amount of chemically enriched matter is essential, as well as the geometry and kinematics of the circumstellar material. The goal of the present paper is, therefore, to extract these based on a detailed study of CO-band emission in medium- and high-resolution $K$-band spectra of the Galactic B[e]SG star GG Car.

\section{Observation and data reduction}

A medium-resolution $(R=4500) K$-band $(1.95-2.45 \mu \mathrm{m}) \mathrm{spec}$ trum for the Galactic B[e] star GGCar was obtained on 2012 January 9, using the Spectrograph for INtegral Field Observation in the Near-Infrared (SINFONI; Eisenhauer et al. 2003; Bonnet et al. 2004) on the VLT UT4 telescope. The observations were performed with an $\mathrm{AB}$ nod pattern and an $8 \times 8 \operatorname{arcsec}^{2}$ field of view. For telluric correction and flux calibration, a B-type standard star was observed at similar airmass. The data were reduced with the SINFONI pipeline (version 2.2.9).

For flux calibration, we applied a Kurucz model (Kurucz 1993) to the standard star spectrum and scaled it to its Two Micron All-Sky Survey (2MASS, Skrutskie et al. 2006) $K_{\mathrm{S}}$-band magnitude. The signal-to-noise ratio $(\mathrm{S} / \mathrm{N})$ of the final spectrum is $\sim 60$. The heliocentric velocity correction was carried out and the spectrum was dereddened with the extinction value listed in Table 1 applying the interstellar reddening law of Howarth (1983).

A high-resolution spectrum $(R \simeq 50000)$ in the $K$-band $(2.276-2.326 \mu \mathrm{m})$ was obtained on 2009 December 2 using the CRyogenic high-resolution InfraRed Echelle Spectrograph (CRIRES; Kaeufel et al. 2004). The observations were performed with a slit-width of 0.4 arcsec, and a standard nodding on-slit strategy was applied to remove sky- and detector glow. For telluric corrections, a standard star was observed close in airmass and directly after the science object. The data reduction was performed with the CRIRES pipeline. The final spectrum was corrected for heliocentric velocity, and it has an $\mathrm{S} / \mathrm{N}$ of $\sim 120$.

\section{Results}

In addition to the emission from both the ${ }^{12} \mathrm{CO}$ and ${ }^{13} \mathrm{CO}$ bands, the SINFONI spectrum (Fig. 1) clearly shows emission lines from the hydrogen Pfund series. To model their contribution, we first adopted a Gaussian-like profile, indicative of an origin within a wind or shell. However, no reasonable fit of their profile shape could be achieved.

Double-peaked lines have been identified in the optical spectra of GGCar, e.g., forbidden emission lines of [OI] and [Ca II] (Muratore et al. 2012) and permitted emission lines of Fe II (Marchiano et al. 2012). Furthermore, emission lines from the hydrogen Balmer and Paschen series appear to be doublepeaked, typically with the red peak more intense than the blue one (Marchiano et al. 2012). Whether the Pfund lines show the same behavior is hard to tell because of the poor quality of the SINFONI spectrum (see noise level shortward of $2.29 \mu \mathrm{m}$ ).

Pfund emission is usually formed within a narrow region. Considering that the line profiles could be double-peaked, the emission might arise from a (narrow) ring within the rotating disk. The spectral resolution of SINFONI, $v_{\text {res }} \simeq 60 \mathrm{~km} \mathrm{~s}^{-1}$, smears out the possibly double-peaked profile shape so that we cannot measure the proper rotation velocity from the peak separation. The rotation speed, projected to the line of sight, was therefore obtained from fitting the line widths with a rotation profile convolved to the proper spectral resolution. The optimum fit was achieved for $v_{\text {rot,Pf }} \sin i=(94 \pm 2) \mathrm{km} \mathrm{s}^{-1}$.

The Pfund series reaches its edge (i.e., the Pfund discontinuity) blueward of the $\mathrm{CO}$ band head. Furthermore, the higher the transition, the shorter the wavelength separation between individual lines so that the lines start to blend. The broad profiles of the Pfund lines in our spectra together with the low spectral resolution result in the formation of a quasi-continuum of the higher transitions of the Pfund series, i.e., in the wavelength region $\lambda \lesssim 2.325 \mu \mathrm{m}$ and extending blueward of the CO band head (see, e.g., Kraus et al. 2000). However, the SINFONI spectrum does not show any indication for such a quasi-continuum on the short wavelength edge of the $\mathrm{CO}$ spectrum, so the number of Pfund lines in the spectrum must be limited. This agrees with the fact that population of the higher levels in especially high-density media is usually prevented by pressure ionization effects. The maximum Pfund transition cannot be determined purely based on the SINFONI data, but the highest Pfund line should occur redward of the $\mathrm{CO}$ band edge.

The CRIRES spectrum (Fig. 2) shows the fully resolved structure of the first ${ }^{12} \mathrm{CO}$ band head emission. While fitting this short portion of the emission spectrum is not sensitive to the 
M. Kraus et al.: Molecular emission from GG Carinae's circumbinary disk

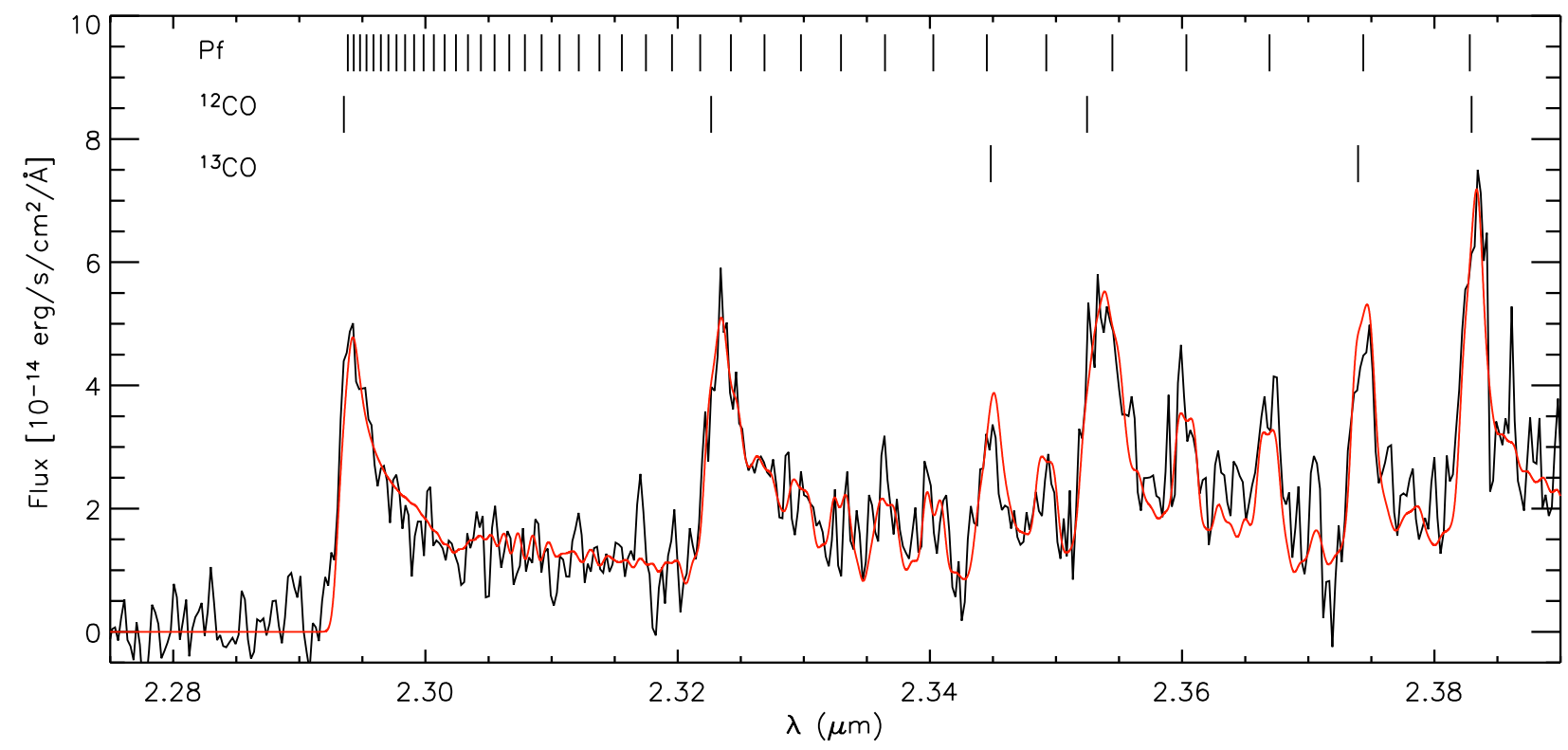

Fig. 1. Fit (red/gray) to the flux-calibrated, continuum-subtracted SINFONI spectrum (black) of GG Car. Positions of the CO band heads and hydrogen Pfund lines are indicated.

CO temperature and column density, it delivers detailed knowledge of the $\mathrm{CO}$ kinematics. The blue shoulder and red peak are typical characteristics for emission in a rotating ring or disk (e.g., Carr et al. 1993; Carr 1995; Najita et al. 1996; Kraus et al. 2000), and their separation (as shown by the arrow in the top panel of Fig. 2) directly determines the disk rotational velocity, projected to the line of sight, in the CO-forming region.

The observed band head characteristics could also be interpreted with an equatorial outflow in the form of a gaseous ring expanding with constant velocity (e.g., Kraus et al. 2010), as the resulting double-peaked profile is indistinguishable from the rotating one. Nevertheless, we consider here the scenario of a rotating disk as more reliable based on the studies of Pereyra et al. (2009).

From our spectrum we obtain $v_{\text {rot,CO }} \sin i=(80 \pm 1) \mathrm{km} \mathrm{s}^{-1}$. This value is lower than that obtained from the width of the Pfund lines and hence perfectly agrees with the scenario of a Keplerian rotating disk in which the CO-emitting region is located farther away than the Pfund-emitting region. From the projected velocity of the $\mathrm{CO}$ gas we obtain the actual rotational velocity of $v_{\text {rot,CO }}=(91.5 \pm 7.5) \mathrm{km} \mathrm{s}^{-1}$, assuming that the disk is co-planar with the orbital orientation.

For the model computations, we made use of the $\mathrm{CO}$ disk code developed by Kraus et al. (2000), modified to account for the emission of ${ }^{13} \mathrm{CO}$ (Kraus 2009; Oksala et al., in prep.). Former calculations of $\mathrm{CO}$ emission from a disk revealed that it is sufficient to consider the innermost ring of gas with a constant temperature, column density, and rotation velocity (e.g., Kraus 2009; Liermann et al. 2010; Cidale et al. 2012).

Fitting the CRIRES spectrum with pure $\mathrm{CO}$ emission did not deliver a reasonable fit, because several features of the spectrum could not be satisfactorily reproduced (see top panel of Fig. 2). Therefore, emission from the rotationally broadened Pfund lines was included. The best fit (bottom panel of Fig. 2) was obtained for a cut-off in the Pfund series at the transition Pf 63. The contribution of the Pfund emission is shown in this figure as well.

With this cut-off in the Pfund series and fixing the kinematics in both the $\mathrm{CO}$ and Pfund line emitting regions, we were finally able to obtain reasonable fits to the total SINFONI spectrum
(Fig. 1). The complete set of derived CO disk parameters is listed in Table 2. The last row gives the area of the emitting $\mathrm{CO}$ ring considering the disk inclination and the distance of $d=5 \pm 1 \mathrm{kpc}$ towards GG Car as obtained by Marchiano et al. (2012).

The enrichment in ${ }^{13} \mathrm{C}$ obtained from the strength of the ${ }^{13} \mathrm{CO}$ band emission of GGCar is lower than postulated by Kraus (2009), who suggested ${ }^{12} \mathrm{C} /{ }^{13} \mathrm{C}<10$. This disagreement can be explained by the much lower resolution of the AMBER spectrum in which the strongly rotationally broadened Pfund lines, which are even in the SINFONI spectrum fully resolved only at the long-wavelength end, are completely smeared out. Still, their presence pushes the ${ }^{13} \mathrm{CO}$ band heads up, falsely suggesting a stronger enrichment than the actual value of ${ }^{12} \mathrm{C} /{ }^{13} \mathrm{C}=$ $15 \pm 5$ found with our detailed modeling.

The derived $\mathrm{CO}$ parameters (i.e., temperature and column density) are similar to those found for other B[e]SGs such as LHA 120-S 12 and LHA 120-S 73 in the Large Magellanic Cloud (Liermann et al. 2010), LHA 115-S65 in the Small Magellanic Cloud (Oksala et al. 2012), and CPD-529243 (Cidale et al. 2012) and MWC 349A (Kraus et al. 2000) in the Galaxy. Only one Galactic object, HD 327083, seems to be extreme in the sense that its disk, studied by Wheelwright et al. (2012), has the coolest $\left(T_{\mathrm{CO}}=1721 \pm 540 \mathrm{~K}\right)$ and densest $\left(N_{\mathrm{CO}}=(0.4 \pm 1.8) \times 10^{24} \mathrm{~cm}^{-2}\right) \mathrm{CO}$ gas. However, in the case of HD 327083 only the first band head was studied, which is a poor tracer for density and temperature. These parameters can be determined with a high precision only from a complete $\mathrm{CO}$ band covering three or more band heads.

\section{Discussion}

\subsection{Circumbinary disk of GG Car}

The binary parameters derived by Marchiano et al. (2012) resulted in a size of the semi-major axis of the system (corrected for inclination) of $A=0.65 \pm 0.04 \mathrm{AU}$ and individual stellar masses of $M_{\text {prim }} \simeq 26 \pm 4 M_{\odot}$ and $M_{\text {sec }} \simeq 12 \pm 2 M_{\odot}$. Interpreting the velocity of the $\mathrm{CO}$ gas with rotation in a Keplerian disk, the distance of the CO emitting ring would be $R_{\mathrm{CO}}=2.88 \pm 0.89 \mathrm{AU}$ when orbiting the primary, and $R_{\mathrm{CO}}=1.35 \pm 0.45 \mathrm{AU}$ when 


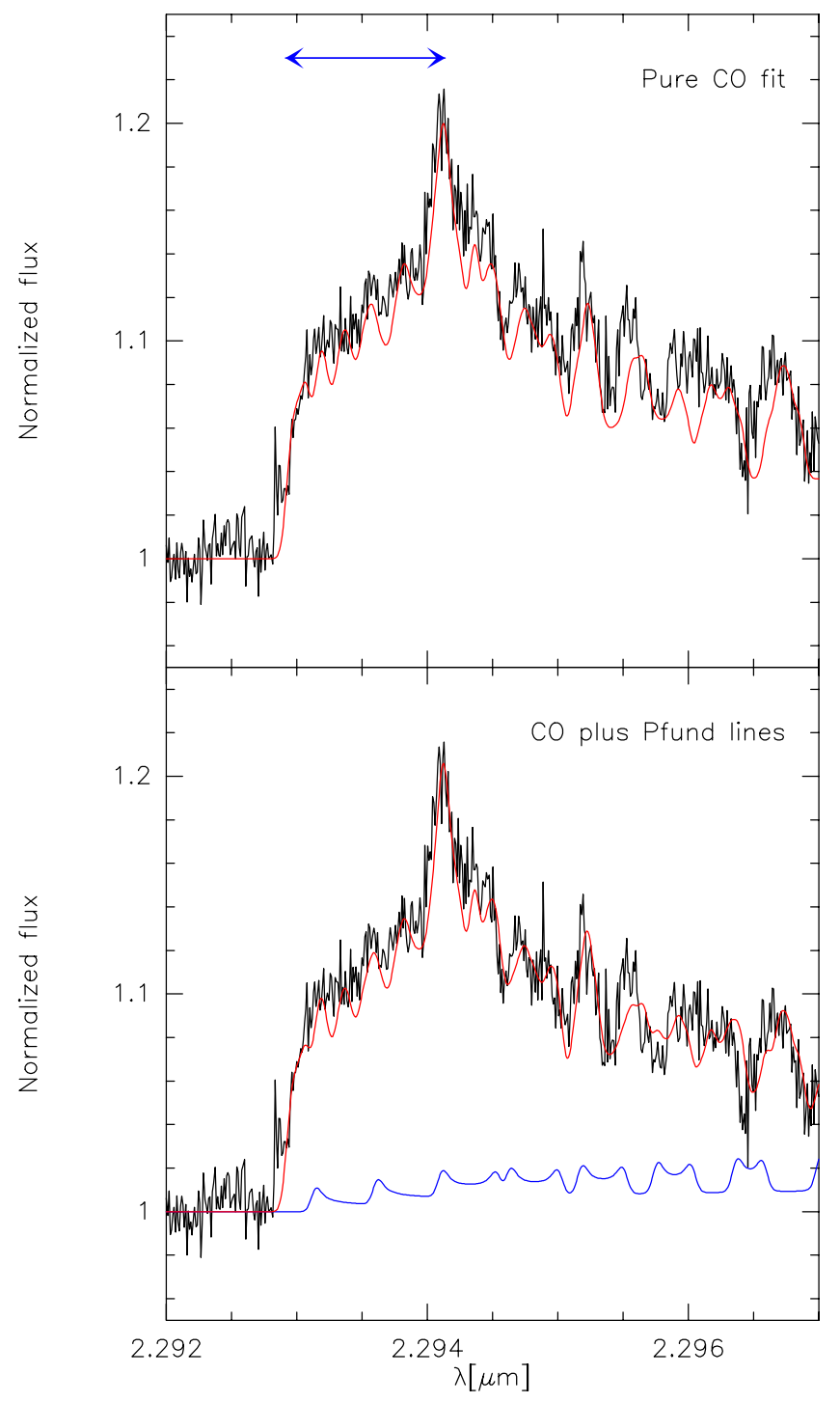

Fig. 2. Fit (red/gray) to the observed, normalized CRIRES spectrum of GGCar (black) considering the emission to consist of either pure $\mathrm{CO}$ band (top panel) or $\mathrm{CO}$ band plus Pfund line emission (bottom panel).

Table 2. Parameters of the CO disk.

\begin{tabular}{ll}
\hline \hline$T_{\mathrm{CO}}[\mathrm{K}]$ & $3200 \pm 300$ \\
$N_{\mathrm{CO}}\left[\mathrm{cm}^{-2}\right]$ & $(5 \pm 3) \times 10^{21}$ \\
$i\left[{ }^{\circ}\right]$ & $63 \pm 9$ \\
${ }^{12} \mathrm{C} /{ }^{13} \mathrm{C}$ & $15 \pm 5$ \\
$v_{\mathrm{rot}, \mathrm{CO}}\left[\mathrm{km} \mathrm{s}^{-1}\right]$ & $91.5 \pm 7.5$ \\
$v_{\mathrm{rot}, \mathrm{Pf}}\left[\mathrm{km} \mathrm{s}^{-1}\right]$ & $108 \pm 11$ \\
$A_{\mathrm{CO}}\left[\mathrm{AU}^{2}\right]$ & $1.33 \pm 0.73$ \\
\hline
\end{tabular}

Notes. The inclination angle is taken from Marchiano et al. (2012).

orbiting the secondary star. In both cases, this distance is larger than the binary separation. The CO must therefore be located in a circumbinary disk orbiting a central mass of $M_{\text {binary }} \simeq 38 \pm 6 M_{\odot}$.

Figure 3 displays the Keplerian velocity as a function of distance for the minimum and maximum binary masses. The horizontal lines show the range in rotation velocity obtained from modeling the CRIRES data, and the intersections deliver the range of possible distances of the circumbinary $\mathrm{CO}$-emitting ring from the center of gravity. From the size of the emitting

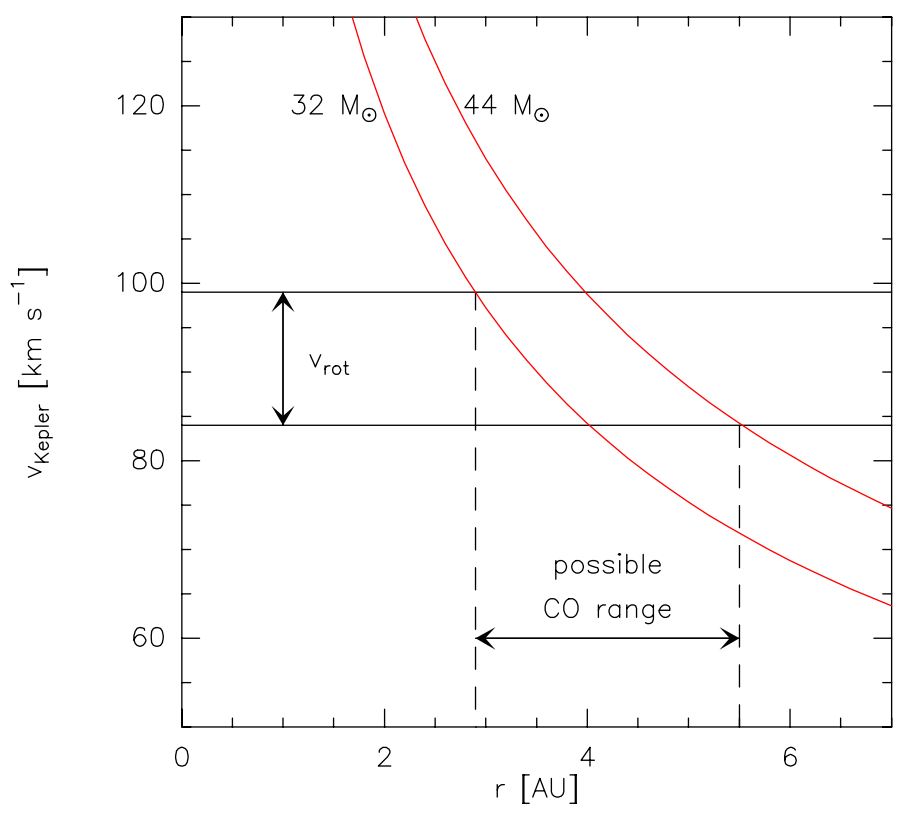

Fig. 3. Keplerian velocity distribution considering the total minimum and maximum binary mass. Horizontal lines represent the range in rotational velocity of the $\mathrm{CO}$ gas. The location range of the $\mathrm{CO}$ ring is marked (dashed lines).

$\mathrm{CO}$ area (Table 2) the outer edge, $R_{\text {out }}$, of the $\mathrm{CO}$ emitting ring can be calculated as a function of its inner edge, $R_{\text {in }}$. Considering the error in the $\mathrm{CO}$ area, we computed the two limiting scenarios and obtained sizes for the CO ring that range from $R_{\text {out }}=$ $2.97 \pm 0.04 \mathrm{AU}$ for the minimum inner edge of $R_{\text {in,min }}=2.90 \mathrm{AU}$, and $R_{\text {out }}=5.54 \pm 0.02 \mathrm{AU}$ for the maximum inner edge of $R_{\text {in, } \max }=5.50 \mathrm{AU}$, indicating that the emitting $\mathrm{CO}$ gas is confined in a narrow ring. Considering even the largest distance, the CO-emitting ring falls well inside the value of 230 stellar radii ( $\sim 34 \mathrm{AU}$ for a primary stellar radius of $32 R_{\odot}$ ) suggested by Marchiano et al. (2012) as the maximum possible distance of the CO-emitting region based on temperature arguments.

The B[e]SG star HD 327083 was also recently found to have a circumbinary rotating disk displaying strong $\mathrm{CO}$ band emission (Wheelwright et al. 2012). In both this and the GG Car system, the minimum inner edge of the $\mathrm{CO}$ ring is located at a similar distance of $\sim 3 \mathrm{AU}$. Other interesting objects in which a close (in projection) binary component was discovered by means of interferometry are the A[e] supergiant HD 62623 (Millour et al. 2011, although this star was recently re-classified as a B8-9 IbII star by Borges Fernandes et al., in prep.) and the B[e]SG candidates HD 87643 (Millour et al. 2009) and V921 Scorpii (=CD-42 11721; Kraus et al. 2012). Only the first two have been found to display $\mathrm{CO}$ band emission, and detailed modeling will determine the kinematics of the $\mathrm{CO}$ gas and possibly help to distinguish between the scenarios of circumstellar or circumbinary origin (Muratore et al., in prep.).

\subsection{Evolutionary stage of GG Car}

The origin of the $\mathrm{B}[\mathrm{e}]$ phenomenon in massive evolved stars like $\mathrm{B}$ [e]SGs is still not fully resolved. A disk or ring of high-density material containing gas and dust is usually seen around these objects, but the disk formation mechanism is not fully understood and the disk kinematics have not been sufficiently studied yet. Because post-main sequence massive stars possess strong linedriven stellar winds during their main-sequence evolution, these 


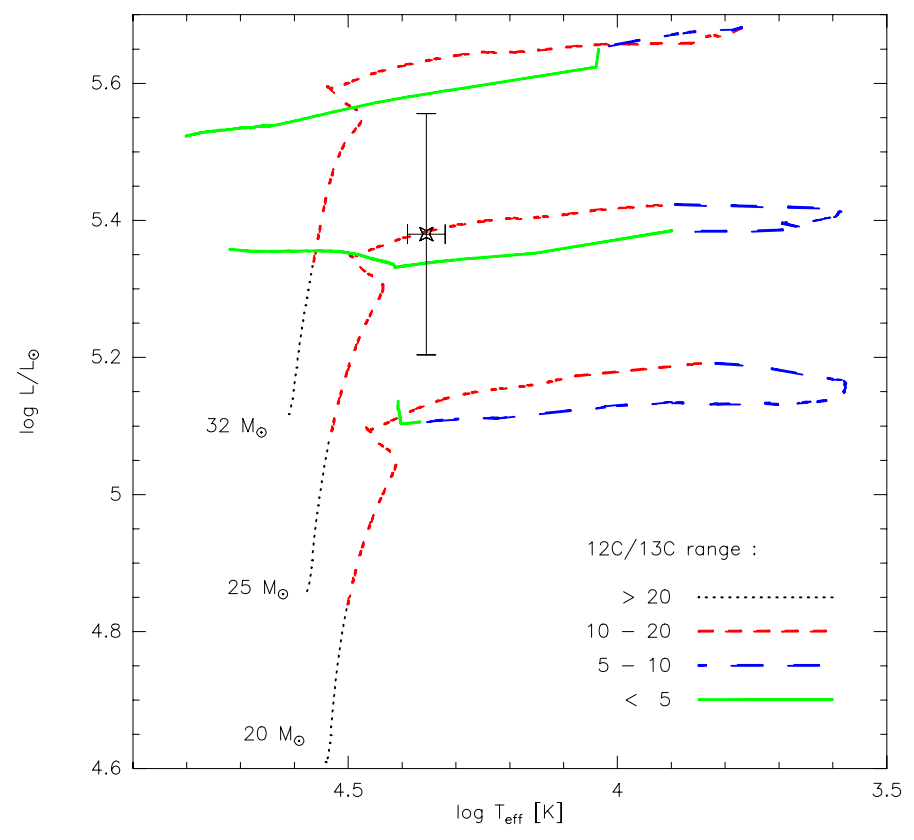

Fig. 4. HR diagram showing the position of the primary component of GG Car with respect to new evolutionary tracks of Ekström et al. (2012) for stars at solar metallicity rotating initially with $v_{\text {ini }} / v_{\text {crit }}=0.4$. The enrichment in ${ }^{13} \mathrm{C}$ over the course of stellar evolution is indicated.

disks cannot be remnants from their pre-main sequence stage, but likely formed from highly non-spherically symmetric stellar mass-loss. A possible scenario discussed in the literature to explain the formation of outflowing high-density disks in singlestar evolution is the bistability mechanism induced by rotation (Lamers \& Pauldrach 1991; Pelupessy et al. 2000). However, the density of these disks increases enormously when the slowwind solution (Curé 2004) at high rotation rates $(\Omega>0.6)$ is considered (Curé et al. 2005). In both, the rapid stellar rotation is an important prerequisite, but it is even more important to know if a massive main-sequence star could remain close to the limit of critical rotation for a substantial fraction of its lifetime (Langer 1998). Rotation velocities at a substantial fraction of their critical values have been found so far for two B[e]SGs LHA 115-S 23 (Kraus et al. 2008) and LHA 115-S 65 (Zickgraf 2000; Kraus et al. 2010) in the Small Magellanic Cloud. But whether all $\mathrm{B}[\mathrm{e}] \mathrm{SGs}$ are indeed rapid rotators is not known.

Other scenarios consider that binary interaction up to full merging of massive binaries can cause the $\mathrm{B}[\mathrm{e}]$ phenomenon in the supergiants (Langer \& Heger 1998; Podsiadlowski et al. 2006). However, the binary merger scenario seems to hold so far for only one object of this class, i.e., the B[e]SG R4 in the Small Magellanic Cloud.

The appearance of the $\mathrm{B}[\mathrm{e}]$ phenomenon in the eccentric binary system GG Car might also be linked to binary interaction, at least to some extent, and in the following we briefly discuss the evolutionary stage of the primary component of GG Car and its possible evolutionary history.

Figure 4 shows the location of the primary component of GG Car in the HR diagram based on its effective temperature and luminosity (Table 1). Also shown are new tracks from Ekström et al. (2012) for single-star evolution at solar metallicity and initial rotation speeds of about $40 \%$ of the critical velocity. The rotation speed might not be fully appropriate for GG Car, for which no rotational velocity could be obtained so far due to the lack in photospheric lines. But an initial rotation speed like this has been found as the peak of the velocity distribution for young B stars (Huang et al. 2010), and thus can be regarded as representative for the general behavior of rotating stars. The different line styles used along the evolutionary tracks in Fig. 4 mark regions with different values of the ${ }^{12} \mathrm{C} /{ }^{13} \mathrm{C}$ surface abundance ratio. This ratio starts with an initial (interstellar) value of $\sim 90$ on the zero-age main sequence, and drops to a final value of $\$ 5$ at late, post-red supergiant evolutionary phases, tracing a strong enrichment in ${ }^{13} \mathrm{C}$ during the evolution of massive stars. The value of the carbon isotopic ratio found for the circumbinary material of GG Car is ${ }^{12} \mathrm{C} /{ }^{13} \mathrm{C}=15 \pm 5$. This high value excludes a late (i.e., postred supergiant) evolutionary phase, but excellently agrees with the primary having just evolved beyond the main sequence (see Fig. 4) considering that the primary has evolved unaffected by its companion.

The initial mass and current location of GGCar in the HR diagram is very similar to another $\mathrm{B}[\mathrm{e}] \mathrm{SG}$, the object LHA 120-S 12 in the Large Magellanic Cloud. Furthermore, the $\mathrm{CO}$ ring around this star was found to have very similar $\mathrm{CO}$ column density $\left(N_{\mathrm{CO}}=(2.5 \pm 0.5) \times 10^{21} \mathrm{~cm}^{-2}\right)$ and temperature $(2800 \pm 500 \mathrm{~K})$, and its carbon isotopic ratio was determined to $20 \pm 2$ (Liermann et al. 2010). Like LHA 120-S 12, GG Car is hence another example of a B[e]SG with only slight enhancement of ${ }^{13} \mathrm{C}$ and consequently is in a pre-red supergiant phase, while other objects were found to show strong ${ }^{13} \mathrm{C}$ enrichment, which classifies them as post-red supergiants or post-yellow hypergiants (see e.g., Liermann et al. 2010; Muratore et al. 2010; Oksala et al., in prep.).

Although the carbon isotopic ratio is the strongest indicator, there are additional arguments in favor of an evolutionary phase of GGCar just beyond the main sequence. Unlike the well-known LBVs HR Car and AGCar, no nebulosity was detected around GG Car (Thackeray 1950). Therefore, it seems less evolved than its close-by neighbors. Furthermore, assuming that the three stars HR Car, AG Car and GG Car have similar ages (based on similar sky location and distance), it is clear that the more massive stars are more evolved. Comparing the ages of AGCar and HR Car with the evolutionary tracks of stars in the proper mass range for the primary of GGCar delivers an evolutionary stage just beyond the main sequence, in perfect agreement with its position in the HR diagram.

In summary, all evidence $\left({ }^{13} \mathrm{C}\right.$ enrichment, age, missing LBV nebulosity) seems to speak in favor of a single-star evolutionary scenario for the primary component of GG Car, which is currently in an early post-main sequence evolutionary phase, according to the HR diagram.

\subsection{Origin of the circumbinary disk}

One question that remains to be discussed concerns the origin of the cool and dense circumbinary disk seen in both $\mathrm{CO}$ and dust emission. The transportation and accumulation of stellar material into circumbinary orbit might have happened in two different ways, either via non-conservative Roche lobe overflow occurring earlier in the evolution of the primary, or the primary became a Be star during its late stages of main-sequence evolution. In the following we briefly describe and discuss both options.

Roche lobe overflow results when the stellar radius becomes larger than the size of its Roche lobe. This can happen at different stages of stellar evolution. To compute the effective radius, $r_{1}$, of GG Car's Roche lobe we applied the equation

$$
\frac{r_{1}}{A}=\frac{0.49 q^{2 / 3}}{0.6 q^{2 / 3}+\ln \left(1+q^{1 / 3}\right)}
$$


provided by Eggleton (1983). The parameter $q$ is the mass ratio and $A$ is the binary separation. This equation is strictly valid for circular orbits only. In eccentric binaries, the mass transfer will take place when the two stars are closest to each other, i.e., during periastron passage. We can hence approximate an equivalent Roche lobe for eccentric binaries by replacing the binary separation $A$ by the periastron distance $A_{\text {peri }}=A(1-e)$, where $e$ is the eccentricity. The behavior of $r_{1} / A_{\text {peri }}$ as a function of the mass ratio $q$ is shown in the right panel of Fig. 5. For the chosen range, the radius of the Roche lobe increases with increasing $q$. The mass ratio (including its errors) of GG Car and the resulting size of the effective Roche lobe is indicated.

To determine if the radius of GG Car's primary star could have filled its Roche lobe during its past evolution, we computed the evolution of the stellar radius during and beyond the main sequence for stars with different appropriate initial masses. Assuming that the periastron distance remained constant during the star's evolution, we normalized the stellar radii to GG Car's current periastron distance and plot them versus the evolution of the stellar effective temperature in the left panel of Fig. 5. The location of GG Car's primary is included in the plot, as well as the range in Roche lobe radii for the current mass ratio. Obviously, as long as neither the binary separation nor the mass ratio has changed during earlier evolution, the primary of GGCar has not filled its Roche lobe yet. If, however, we postulate that the circumbinary material originates from non-conservative Roche lobe overflow in the past, then the original mass ratio must have been higher than the current value. A higher mass ratio, on the other hand, increases the effective radius of the Roche lobe. Hence, for mass transfer to happen, the original periastron distance must have been (much) smaller than it is today, and the mass transfer must have resulted in widening the system.

While this scenario cannot be excluded (see, e.g., Sepinsky et al. 2007, 2009), non-conservative mass transfer usually leads to a shrinking orbit. In addition, standard theory of binary evolution assumes that the originally eccentric orbit circularizes immediately after the onset of mass transfer (e.g., Hurley et al. 2002). This assumption seems to be justified because only few eccentric evolved binary systems are observed (e.g., de Mink et al. 2007). In this context, the eccentric orbit seen for GG Car might speak in favor of an unevolved binary system in which no mass transfer has occurred yet, which would agree with the primary radius being smaller than the effective Roche lobe size during periastron passage (see Fig. 5).

However, Roche lobe overflow cannot be entirely excluded, because Fig. 5 was computed for the mean value of the periastron distance. Taking into account its error, which is based on the error in inclination angle and eccentricity, and considering the extreme case of minimum mass ratio and maximum stellar radius, the effective radius of the Roche lobe drops below the stellar radius. Therefore, Roche lobe overflow could be possible, but would be restricted to an extreme corner in the overall parameter space.

In the second possible scenario, the primary of GG Car could have evolved from a Be star progenitor, particularly if its mass is closer to the lower boundary of the primary's mass range. This value would agree better with those of massive Be stars found close to the end of and slightly beyond the main sequence. Be stars show Balmer emission that is supposedly arising from a geometrically thin, ionized gaseous disk in Keplerian rotation (e.g., Porter \& Rivinius 2003). The disk is thought to be formed by mass loss through an equatorial, viscous decretion disk (Lee et al. 1991; Porter 1999; Krtička et al. 2011) triggered by the

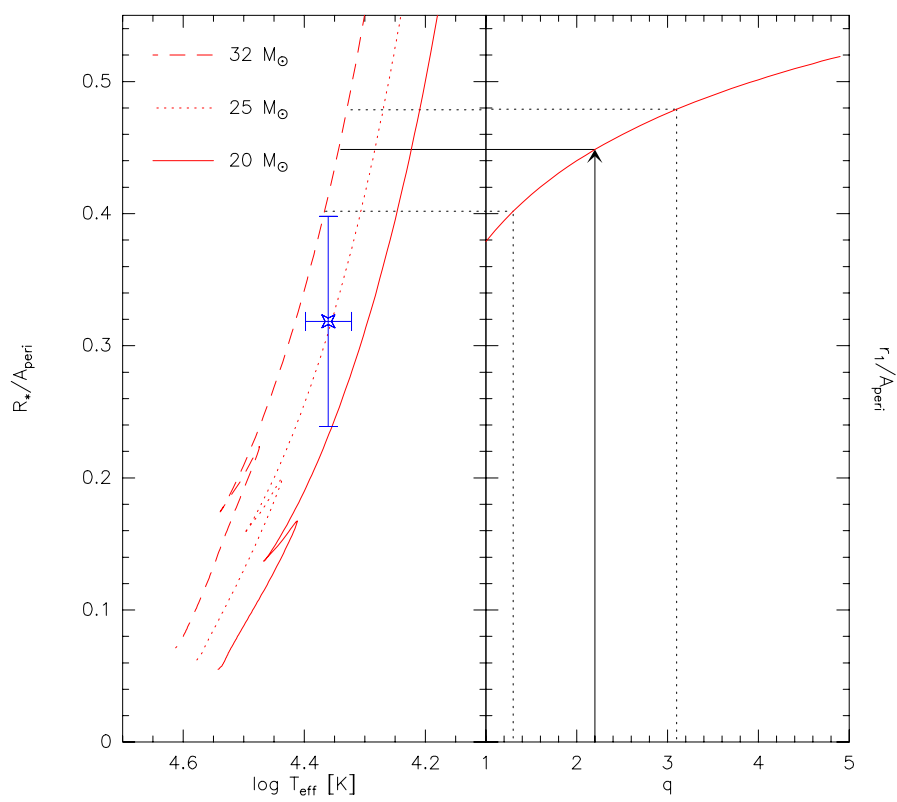

Fig. 5. Left panel: stellar radius, normalized to the minimum binary separation of the GG Car system, during the main and post-main sequence evolution of stars with different initial masses, plotted in terms of the effective temperature variation. Right panel: relative effective Roche lobe radius for a range of mass ratios. The value (with errors) for GG Car is marked.

close to critical rotation of the star (e.g., Townsend et al. 2004). Typical sizes range from a few up to a few hundred stellar radii.

Assuming that the plane of the disk around the primary is coplanar with the binary orbit, this disk can cause mass loss into circumbinary orbits in two ways. The binary separation of GG Car during periastron is $A_{\text {peri }}=0.468 \pm 0.048 \mathrm{AU}$, which corresponds to roughly 5-15 times the stellar radius during the main sequence evolution (see Fig. 5). Therefore, during periastron passage even a small disk will extend beyond the Roche radius and disk mass could stream to the companion through the Lagrangian point and/or leave the Roche surface to accumulate in circumbinary orbits. On the other hand, the secondary will have to pass through the disk as well, and disk material approaching the star from behind will be accelerated and hence kicked out of the system.

It is hard to tell which of the two suggested scenarios is real. But given the need for an extreme parameter combination for Roche lobe overflow to work, in combination with the eccentric orbit of GG Car that points to an unevolved binary system, the Be star scenario might be slighlty more favorable. Nevertheless, only proper theoretical modeling of both can give a more conclusive answer to the question of the evolutionary history of GG Car. In addition, a proper study of the kinematics of the circumstellar/circumbinary material needs to be performed based on many individual tracers of different regions. The easiest indicators for kinematics are certainly the forbidden emission lines. Among the observed lines are [OI] and [Ca II], which are the most reliable tracers for the high-density disk regions (Aret et al. 2012). The profiles of the [OI] lines, which seem to consist of two distinct double-peaked profiles (Muratore et al. 2012), already show that the distribution and kinematics of the material in the GG Car system is highly complex. High-quality observations, especially over the full orbital period, are of vital importance to extract a more complete picture of this fascinating object. 


\section{Conclusions}

We presented our analysis of the CO-band emission of the eccentric binary system GG Car. CO bands are detected in both medium- and high-resolution $K$-band spectra obtained with the SINFONI and CRIRES spectrographs. The SINFONI spectrum extends over the full CO-band structure, which gives insight into the density $\left(N_{\mathrm{CO}}=(5 \pm 3) \times 10^{21} \mathrm{~cm}^{-2}\right)$ and temperature $\left(T_{\mathrm{CO}}=3200 \pm 500 \mathrm{~K}\right)$ of the emitting region, which we find to be confined in a narrow circumbinary ring. In addition, the SINFONI spectrum covers the band heads from the isotopic molecule ${ }^{13} \mathrm{CO}$, allowing us to determine the evolutionary phase and hence the age of the star at the time of the material ejection. Our modeling derives a value ${ }^{12} \mathrm{C} /{ }^{13} \mathrm{C}=15 \pm 5$, confirming a slightly evolved (supergiant) nature of GG Car's primary component. The CRIRES spectrum contains the spectrally resolved structure of the first ${ }^{12} \mathrm{CO}$ band head. From its shape we find that the motion of the $\mathrm{CO}$ gas is consistent with Keplerian rotation with a velocity, projected to the line of sight, of $80 \pm 1 \mathrm{~km} \mathrm{~s}^{-1}$.

Based on these results, we discussed the origin of the circumbinary disk in this eccentric binary system. In particular, two scenarios were taken into consideration. The first one considered the possibility that the primary component underwent non-conservative Roche lobe overflow during its past evolution, while the second considered that the mass transport and accumulation on circumbinary orbits could have happened during Be phases of the primary component. We cannot firmly prove or disprove the validity of either scenario. However, the conditions for non-conservative Roche lobe overflow are only met in an extreme corner of the parameter space. Therefore, slight preference is given to the suggestion that during late phases of its main-sequence evolution, GG Car's primary could have been a classical Be star.

Acknowledgements. We thank the anonymous referee for the valuable comments on the manuscript. This research made use of the NASA Astrophysics Data System (ADS). M.K., M.E.O., D.H.N., and A.A. acknowledge financial support from GAČR under grant number P209/11/1198. The Astronomical Institute Ondřejov is supported by the project RVO:67985815. Financial support for International Cooperation of the Czech Republic (MŠMT, 7AMB12AR021) and Argentina (Mincyt-Meys, ARC/11/10) is acknowledged. M.F.M. is a research fellow of the Universidad Nacional de La Plata, Argentina. M.B.F. acknowledges Conselho Nacional de Desenvolvimento Cientifico e Tecnológico (CNPq-Brazil) for a post-doctoral grant. A.A. acknowledges financial support from the research project SF0060030s08 of the Estonian Ministry of Education and Research. L.C. acknowledges financial support from the Agencia de Promoción Científica y Tecnológica (BID 1728 OC/AR PICT 885), PIP 0300 CONICET, and the Programa de Incentivos G11/109 of the Universidad Nacional de La Plata, Argentina.

\section{References}

Allen, D. A. 1973, MNRAS, 161, 145

Aret, A., Kraus, M., Muratore. M. F., \& Borges Fernandes, M. 2012, MNRAS, 423, 284

Barbier, R., \& Swings, J. P. 1982, in Be stars (Dordrecht: D. Reidel Publishing Co.), IAU Symp., 98, 103

Bonnet, H., Abuter, R., Baker, A., et al. 2004, The Messenger, 117, 17

Borges Fernandes, M. 2010, Rev. Mex. A\&A Ser. Conf., 38, 98

Bouchet, P., \& Swings, J. P. 1982, in Be stars (Dordrecht: D. Reidel Publishing Co.), IAU Symp., 98, 241

Brandi, E., Gosset, E., \& Swings, J.-P. 1987, A\&A, 175, 151

Carr, J. S. 1995, Ap \& SS 224, 25

Carr, J. S., Tokunaga, A. T., Najita, J., Shu, F. H., \& Glassgold, A. E. 1993, ApJ, 411, L 37

Cidale, L. S., Borges Fernandes, M., Andruchow, I., et al. 2012, A\&A, 548, A72 Cohen, M., \& Barlow, M. J. 1983, ApJ, 238, 585

Curé, M. 2004, ApJ, 614, 929
Curé, M., Rial, D. F., \& Cidale, L. 2005, A\&A, 437, 929

de Mink, S. E., Pols, O. R., \& Hilditch, R. W. 2007, A\&A, 467, 1181

Eggleton, P. P. 1983, ApJ, 268, 368

Eisenhauer, F., Abuter, R., Bickert, K., et al. 2003, in Proc. SPIE, 4841, 1548

Ekström, S., Georgy, C., Eggenberger, P., et al. 2012, A\&A, 537, A146

Gnedin, Y. N., Kiselev, N. N., Pogodin, M. A., Rosenbush, A. E., \& Rosenbush,

V. K. 1992, Soviet Ast. Lett., 18, 182

Gosset, E., Surdej, J., \& Swings, J.-P. 1984, A\&AS, 55, 411

Gosset, E., Hutsemékers, D., Surdej, J., \& Swings, J.-P. 1985, A\&A, 153, 71

Hernández, C. A., López, L., Sahade, J., \& Thackeray, A. D. 1981, PASP, 93, 747

Howarth, I. D. 1983, MNRAS, 203, 301

Huang, W., Gies, D. R., \& McSwain, M. V. 2010, ApJ, 722, 605

Humphreys, R. M., Lamers, H. J. G. L. M., Hoekzema, N., \& Cassatella, A. 1989, A\&A, 218, L17

Hurley, J. R., Tout, C. A., \& Pols, O. R. 2002, MNRAS, 329, 897

Hutsemékers, D., \& Van Drom, E. 1991, A\&A, 248, 141

Kaeufl, H.-U., Ballester, P., Biereichel, P., et al. 2004, in Proc. SPIE, 5492, 1218

Klare, G., \& Neckel, T. 1977, A\&AS, 27, 215

Kraus, M. 2009, A\&A, 494, 253

Kraus, M., Krügel, E., Thum, C., \& Geballe, T. R. 2000, A\&A, 362, 158

Kraus, M., Borges Fernandes, M., Kubát, J., \& de Araújo, F. X. 2008, A\&A, 487, 697

Kraus, M., Borges Fernandes, M., \& de Araújo, F. X. 2010, A\&A, 517, A30

Kraus, S., Calvet, N., Hartmann, L., et al. 2012, ApJ, 746, L2

Krtička, J., Owocki, S. P., \& Meynet, G. 2011, A\&A, 527, A84

Kurucz, R. L. 1993, VizieR Online Data Catalog, VI/039

Lamers, H. J. G. L. M., \& Pauldrach, A. W. A. 1991, A\&A, 244, L5

Lamers, H. J. G. L. M., Zickgraf, F.-J., de Winter, D., Houziaux, L., \& Zorec, J. 1998, A\&A, 340, 117

Langer, N. 1998, A\&A, 329, 551

Langer, N., \& Heger, A. 1998, in B[e] Stars, eds. A. M. Hubert, \& C. Jaschek (Dordrecht, Boston: Kluwer Academic Publishers), Astrophys. Space Sci. Lib., 233, 235

Lee, U., Osaki, Y., \& Saio, H. 1991, MNRAS, 250, 432

Liermann, A., Kraus, M., Schnurr, O., \& Borges Fernandes, M. 2010, MNRAS, 408, L 6

Lopes, D. F., Damineli Neto, A., \& de Freitas Pacheco, J. A. 1992, A\&A, 482

Machado, M. A., de Araújo, F. X., de Faria Lopes, D., \& Pereira, C. B. 2004 Rev. Mex. A\&A Ser. Conf., 20, 239

Marchiano, P., Brandi, E., Muratore, M. F., et al. 2012, A\&A, 540, A91

McGregor, P. J., Hyland, A. R., \& Hillier, D. J. 1988, ApJ, 324, 1071

Millour, F., Chesneau, O., Borges Fernandes, M., et al. 2009, A\&A, 507, 317

Millour, F., Meilland, A., Chesneau, O., et al. 2011, A\&A, 526, A107

Morris, P. W., Eenens, P. R. J., Hanson, M. M., Conti, P. S., \& Blum, R. D. 1996, ApJ, 470, 597

Muratore, M. F., Kraus, M., Liermann, A., et al. 2010, BAAA, 53, 123

Muratore, M. F., de Wit, W. J., Kraus, M., et al. 2012, in Circumstellar Dynamics at High Resolution, eds. A. Carciofi, \& T. Rivinius (San Francisco: ASP), ASP Conf. Ser., 464, in press

Najita, J., Carr, J. S., Glassgold, A. E., Shu, F. H., \& Tokunaga, A. T. 1996, ApJ, 462, 919

Oksala, M. E., Kraus, M., Arias, M. L., et al. 2012, MNRAS, 426, L 56

Pelupessy, I., Lamers, H. J. G. L. M., \& Vink, J. S. 2000, A\&A, 359, 695

Pereyra, A., de Araújo, F. X., Magalhães, A. M., Borges Fernandes, M., \& Domiciano de Souza, A. 2009, A\&A, 508, 1337

Pickering, E. C. 1896, Astron. Nachr., 141, 169

Pickering, E. C., \& Fleming, W. P. 1896, ApJ, 4, 142

Podsiadlowski, Ph., Morris, T. S., \& Ivanova, N. 2006, in Stars with the B[e] Phenmenon, eds. M. Kraus, \& A. S. Miroshnichenko (San Francisco: ASP), ASP Conf. Ser., 355, 259

Porter, J. M. 1999, A\&A, 348, 512

Porter, J. M., \& Rivinius, T. 2003, PASP, 115, 1153

Sepinsky, J. F., Willems, B., Kalogera, V., \& Rasio, F. A. 2007, ApJ, 667, 1170

Sepinsky, J. F., Willems, B., Kalogera, V., \& Rasio, F. A. 2009, ApJ, 702, 1387

Skrutskie, M. F., Cutri, R. M., Stiening, R., et al. 2006, AJ, 131, 1163

Swings, J. P. 1974, A\&A, 34, 333

Thackeray, A. D. 1950, MNRAS, 110, 524

Townsend, R. H. D., Owocki, S. P., \& Howarth, I. D. 2004, MNRAS, 350, 189

van Genderen, A. M., Robijn, F. H. A., van Esch, B. P. M., \& Lamers, H. J. G. L. M. 1991, A\&A, 246, 407

Wheelwright, H. E., de Wit, W. J., Weigelt, G., Oudmaijer, R. D., \& Ilee, J. D. 2012, A\&A, 543, A77

Zickgraf, F.-J. 2000, in The Be Phenomenon in early-type stars, eds. M. A. Smith, H. F. Henrichs, \& J. Fabregat (San Francisco: ASP), ASP Conf. Ser., 214,26 